\title{
PREVALENCIA DEL SÍNDROME
} GENITOURINARIO DE LA MENOPAUSIA EN MUJERES E IMPACTO EN LA SEXUALIDAD, EN QUINDÍO (COLOMBIA), 2013-2016

\section{Prevalence of genitourinary syndrome of menopause and impact on sexuality of women in Quindío (Colombia), 2013-2016}

Franklin José Espitia De La Hoz, MD

Recibido: diciembre 14/17 - Aceptado: noviembre 14/18

\section{RESUMEN}

Objetivo: conocer la prevalencia del síndrome genitourinario de la menopausia en una población de mujeres sexualmente activas, con edad igual o mayor a 40 años.

Materiales y métodos: estudio de corte transversal descriptivo. Se incluyeron mujeres diagnosticadas con menopausia, con actividad sexual en las últimas seis semanas, y residentes en el Quindío, departamento ubicado en la zona andina cafetera en Colombia. Se excluyeron mujeres con menopausia quirúrgica, analfabetas, con padecimientos de trastornos mentales o déficit mental. El estudio se realizó en una institución privada de referencia entre el 2013 y 2016. Se hizo un muestreo consecutivo por conveniencia. Se midieron variables sociodemográficas, síntomas vulvo-vaginales y síntomas del tracto urinario inferior relacionados con la presencia del síndrome genitourinario de la menopausia, presencia de disfunción sexual global

1 Servicio de Ginecología y Medicina Materno Fetal, Clínica La Sagrada Familia, Armenia (Colombia). por medio del Índice de Función Sexual Femenina validado en español, y por tipo de disfunción. Se realizó un análisis descriptivo de la información utilizando medidas de frecuencia absoluta y relativa. Se calculó la prevalencia como: número de mujeres con síndrome genitourinario de la menopausia / número de mujeres encuestadas.

Resultados: de un total de 597 mujeres seleccionadas para participar, se analizaron finalmente 558 (93,46\%). La edad promedio fue de 59,73 $\pm 8,4$ años. La prevalencia del síndrome genitourinario de la menopausia fue del 51,61\%. La prevalencia de disfunción sexual en el grupo estudiado fue del $67,2 \%$. Se presentaron trastornos en el deseo $(67,2 \%)$, la excitación (45,16\%), el orgasmo $(44,08 \%)$, la lubricación $(32,25 \%)$ y dolor durante la relación $(13,97 \%)$.

Conclusión: en el grupo de población evaluado se encontró una importante prevalencia del síndrome genitourinario de la menopausia y de disfunción sexual. Se requieren estudios poblacionales para hacer una aproximación a la magnitud del fenómeno en la región latinoamericana. 
Palabras clave: atrofia, dispareunia, menopausia, prevalencia, síntomas del sistema urinario inferior.

\section{ABSTRACT}

Objective: To determine the prevalence of the genitourinary syndrome of menopause in a population of sexually active women 40 years of age or older.

Materials and methods: Descriptive crosssectional study which included women diagnosed with menopause, sexually active over the past six weeks and residents of the Department of Quindío in the coffee growing Andean region of Colombia. Excluded were women with surgical menopause, illiterate, and with mental disorders or deficit. The study was conducted in a private referral centre between 2013 and 2016. Consecutive convenience sampling was used. Measured variables included demographics, vulvovaginal and lower urinary tract symptoms associated with the genitourinary syndrome of menopause, and the presence of global sexual dysfunction, using the Female Sexual Function Index validated in Spanish and by type of dysfunction. A descriptive analysis of the data was performed applying absolute and relative frequency measurements. Prevalence was calculated as the number of women with genitourinary syndrome of menopause/number of women interviewed.

Results: Of a total of 597 women selected to participate in the study, 558 (93.46\%) were included in the final analysis. The prevalence of the genitourinary syndrome of menopause was 51.61\% (62.88\% in women over 55 years of age and 39.32\% in women under 55). The prevalence of sexual dysfunction in the study group was $67.2 \%$. Findings included altered desire (67.2\%), arousal (45.16\%), orgasm (44.08\%), and lubrication (32.25\%), as well as pain during intercourse (13.97\%).

Conclusion: A high prevalence of the genitourinary syndrome of menopause and sexual dysfunction was found in the study population. Population studies are required in order to determine the size of the problem in the Latin American region.
Key words: atrophy, dyspareunia, menopause, prevalence, lower urinary tract symptoms.

\section{INTRODUCCIÓN}

El síndrome genitourinario de la menopausia (SGUM) fue definido en el año 2014 por la Sociedad Internacional para el Estudio de Salud Sexual de las Mujeres y la Sociedad Norteamericana de Menopausia como: "un conjunto de síntomas y signos asociados con una disminución de los estrógenos y otros esteroides sexuales que implican cambios en los labios mayores o menores, clítoris, vestíbulo/ introito, vagina, uretra y vejiga" $(1,2)$. Esta nueva terminología busca reemplazar los términos de vaginitis atrófica/vulvo-vaginitis atrófica o atrofia vulvo-vaginal (AVV) posmenopáusica, ya que son inadecuados y no describen apropiadamente los cambios que acompañan el sistema genitourinario en la menopausia (1). El término vaginitis se asocia a infección, la atrofia tiene implicaciones negativas, y no todas las personas se sienten cómodas hablando de vulva o vagina $(1,3)$. Se ha descrito que aproximadamente el $50 \%$ de las mujeres en la posmenopausia presentan síntomas relacionados con la atrofia urogenital (4). Por otra parte, se ha informado que los cambios del sistema genitourinario en la menopausia se acompañan de trastornos en la función sexual de las mujeres (5).

El diagnóstico del SGUM se hace con base en la sintomatología, las mujeres pueden presentar uno o más síntomas tales como: irritación, picazón, ardor, sequedad y quemazón, acompañados de síntomas urinarios del tipo disuria, frecuencia y urgencia urinaria, o infección del tracto urinario (UTI) recurrente $(1,2)$. El diagnóstico diferencial se debe hacer con la infección vulvo-vaginal (vulvo-vaginitis) o vaginal (vaginitis) y la distrofia vulvar que cursan con sintomatología similar, pero son entidades distintas (2). Para el diagnóstico de las disfunciones sexuales se cuenta con varios instrumentos entre los que está el Índice de Función Sexual Femenina (IFSF), que tiene adecuada confiabilidad, consistencia interna, fiabilidad test-retest y validez discrimi- 
nante (6); además de ser autoadministrado, breve, rápido, sencillo y confiable a la hora de aplicarlo en un amplio rango de edad $(7,8)$, y de haber sido validado en Colombia (8).

El tratamiento del SGUM se orienta a reducir los síntomas, y se basa en cambios en el estilo de vida, aplicación de hormonas locales o sistémicas, el uso de lubricantes y humectantes locales (9). Recientemente se ha descrito el uso de vitamina E local (10), moduladores de los receptores estrogénicos $($ SERM) como el ospemifene $(11,12)$ y el uso de láser vaginal, aunque los estudios realizados muestran resultados a corto plazo (13).

El SGUM ha sido considerado como un problema cada vez más importante por las implicaciones sobre la salud, el bienestar, la sexualidad y la calidad de vida en la mujer que lo padece (3); sin embargo, no hay estudios sobre su prevalencia ni sobre su impacto en la calidad de vida y la sexualidad en la población de mujeres climatéricas en Colombia. Por tanto, el objetivo del presente estudio es hacer una primera aproximación a la prevalencia del síndrome genitourinario de la menopausia en el país, y evaluar su impacto en la sexualidad de las mujeres que ya están en la menopausia.

\section{MATERIALES Y MÉTODOS}

Diseño y población. Estudio de corte transversal descriptivo, realizado del 01 de marzo de 2013 al 30 de septiembre de 2016 en Armenia, capital del departamento del Quindío (ubicado en la región central de Colombia). El estudio fue realizado en la Clínica La Sagrada Familia, institución privada de referencia, de carácter universitario, que atiende a personas pertenecientes a los regímenes de aseguramiento contributivo y subsidiado por el Estado, en el sistema de seguridad social en Colombia. Se incluyeron mujeres con edad mayor o igual a 40 años, diagnosticadas con menopausia, con actividad sexual en las últimas seis semanas y con útero. Se excluyeron mujeres con menopausia quirúrgica, analfabetas, con padecimientos de trastornos mentales o déficit mental. El muestreo fue consecutivo.
Procedimiento. Las pacientes se seleccionaron de la población de mujeres adscritas al programa de menopausia y climaterio de la institución. La recolección de la información estuvo a cargo de las enfermeras, debidamente entrenadas en el proceso de captación de pacientes y diligenciamiento de formularios, y que hacen parte de nuestro equipo de investigación, las cuales encuestaban a las mujeres al momento de ingresar a la consulta con el especialista, previa verificación de los criterios de inclusión y exclusión. A las mujeres que cumplían con los criterios de selección y aceptaron participar en la investigación, se les explicaron los objetivos del estudio, se les solicitó diligenciar el consentimiento informado y se garantizó la confidencialidad del manejo de la información.

Una vez firmado el consentimiento informado se les aplicó un cuestionario de autorreporte en el que se registraron las características sociodemográficas, los síntomas, los antecedentes y los datos del examen clínico. Luego se aplicó el Índice de Función Sexual Femenina (IFSF) para la evaluación de la función sexual. Este instrumento consta de 19 preguntas que evalúan la función sexual femenina en las últimas cuatro semanas. Agrupa seis dominios: Deseo (ítems 1-2), Excitación (ítems 3-6), Lubricación (ítems 7-10), Orgasmo (ítems 11-13), Satisfacción (ítems 14-16) y Dolor (ítems 17-19). Cada pregunta tiene 5 o 6 opciones, con asignación de una puntuación que va de 0 a 5 . El puntaje de cada dominio se multiplica por un factor, y al final el resultado es la suma aritmética de los dominios; a mayor puntaje, mejor sexualidad. El rango total de la puntuación del IFSF va desde 2 hasta 36. Una puntuación menor o igual a 26,55, o cuando la puntuación de algún dominio es menor a 3,6, se considera como criterio de riesgo de disfunción sexual $(6-8,14)$. Posteriormente, el especialista evaluaba la presencia del SGUM de acuerdo con las molestias presentes en la mujer. El diagnóstico se hizo con el hallazgo de uno o más de los siguientes síntomas: vulvo-vaginales (ardor, disminución de la lubricación vaginal, dispareunia, dolor, molestias 
coitales, prurito, quemazón, sangrado poscoital y sequedad vaginal) o del tracto urinario inferior (disuria, tenesmo vesical, nicturia, polaquiuria, infecciones urinarias recidivantes, urgencia urinaria, incontinencia urinaria, infección poscoital y disminución del flujo de orina uretral). Una vez hecho el diagnóstico, a cada mujer se le explicaba la naturaleza de su condición clínica, y se le ofrecían las alternativas terapéuticas (terapia estrógenica local o uso de lubricantes). Las respuestas de cada mujer y los hallazgos del IFSF fueron registrados, tanto en la historia clínica como en un formato especial diseñado por los investigadores.

Variables medidas. Se tuvieron en cuenta variables sociodemográficas tales como: edad, raza, estado civil, ocupación, nivel de estudios, talla, peso, índice de masa corporal (IMC), edad de la menopausia, evolución del tiempo de la menopausia, ingesta de alcohol, tabaquismo, sedentarismo, antecedente personal de depresión o de disfunción sexual, uso de terapia hormonal de reemplazo y antecedente de cáncer de mama; variables de comportamiento sexual como: masturbación, sexo oral, coito -vaginal o anal-, frecuencia promedio de relaciones sexuales mensuales, tiempo de convivencia en pareja, antecedente de abuso sexual o violencia sexual en el matrimonio, pareja con disfunción sexual y enfermedades crónicas. Se indagaron además las preguntas de los dominios de la encuesta IFSF.

Análisis estadístico. Se realizó en el programa estadístico SPSS version 19 y Epi Info version 3.5.1. Las variables categóricas se expresaron como porcentajes. Las variables numéricas se expresaron como media con su desviación estándar. Se calculó la prevalencia de la siguiente manera: número de mujeres con SGUM/número de mujeres encuestadas.

Aspectos éticos. El estudio fue aprobado por la Comisión de ética e investigación de la Clínica La Sagrada Familia. Se solicitó la firma del consentimiento informado para la participación en el estudio, y se garantizó la confidencialidad de la información.

\section{RESULTADOS}

De un total de 663 pacientes inscritas en el programa de climaterio se incluyeron 597 mujeres (90\%), de las cuales trece $(2,17 \%)$ se negaron a participar. De las 584 mujeres restantes que cumplieron los criterios de selección, un total de 14 (2,34\%) no diligenciaron de forma correcta el cuestionario IFSF, otras 12 (2,01\%) se retiraron antes de completar el cuestionario. De este modo, para el análisis final se tomó en cuenta un total de 558 (93,46\%) mujeres.

La edad media de las mujeres incluidas fue de 59,73 (DS $\pm 8,4)$, el IMC fue de 31,8 (DE $\pm 4,65)$, eran mayoritariamente mestizas, amas de casa, tenían unión estable, bajo nivel de escolaridad y pertenecientes al régimen de aseguramiento contributivo. La edad de inicio de la menopausia fue de 48,1 (DS $\pm 5,39$ ), con una duración media de la menopausia de 9,6 (DS \pm 7,5) (tabla 1). El 27,41\% (153) refirió más de 20 años de convivencia en pareja. La práctica sexual más frecuente fue el coito vaginal (100\%), y la menos frecuente el coito anal $(8,42 \%)$; la masturbación es considerada una práctica poco común (5,73\%) en las mujeres encuestadas. El 87,63\% (489) afirmó disfrutar que le practicaran sexo oral. A la pregunta ¿cuántas veces tuvo relaciones sexuales el mes pasado? (periodo definido como el lapso de los treinta días anteriores), el 38,53\% (215) contestó afirmativamente, con una mediana de 3 relaciones sexuales por mes (rango entre $0 \mathrm{y}$ 6). El 96,23\% (537) de las mujeres manifestó que su pareja presentaba alguna disfunción sexual. El 15,05\% (84) informó haber sufrido alguna forma de violencia sexual a lo largo de la vida, mientras que el 19,35\% (108) reportó violencia o abuso sexual por parte de la pareja, luego del inicio de la menopausia, secundario a la negativa de acceder voluntariamente a la actividad sexual.

Se encontró que el 39,24\% de las participantes presentaban HTA, el 7,52\% diabetes tipo 2, el 32,79\% dislipidemias (54,09\% HDL-C bajas, 24,59\% triglicéridos elevados, y 21,31\% colesterol total elevado), y el 6,45\% hipotiroidismo. 


\section{Tabla 1.}

Características sociodemográficas y en salud sexual y reproductiva de la población

de mujeres menopáusicas encuestadas Quindío (Colombia), 2013-2016

$$
\mathrm{n}=\mathbf{5 5 8}
$$

\begin{tabular}{|c|c|}
\hline Variables y categorías & $\mathbf{n}=\%$ \\
\hline Edad* & $59,73 \pm 8,4$ \\
\hline Talla* & $157,91 \pm 8,74$ \\
\hline IMC* & $31,8 \pm 4,65$ \\
\hline \multicolumn{2}{|l|}{ Raza } \\
\hline Hispánicas & $318(56,98 \%)$ \\
\hline Indígenas & $36(6,45 \%)$ \\
\hline Afrocolombianas & $204(36,55 \%)$ \\
\hline \multicolumn{2}{|l|}{ Estado civil } \\
\hline Casada & $219(39,24 \%)$ \\
\hline Unión libre & $117(20,96 \%)$ \\
\hline Soltera & $87(15,59 \%)$ \\
\hline Separada-divorciada & $51(9,13 \%)$ \\
\hline Viuda & $84(15,05 \%)$ \\
\hline \multicolumn{2}{|l|}{ Estrato socioeconómico } \\
\hline Alto & $76(13,62 \%)$ \\
\hline Medio & $423(75,8 \%)$ \\
\hline Bajo & $59(10,57 \%)$ \\
\hline \multicolumn{2}{|l|}{ Ocupación } \\
\hline Amas de casa & $401(71,86 \%)$ \\
\hline Empleadas & $85(15,23 \%)$ \\
\hline Pensionadas & $72(12,9 \%)$ \\
\hline \multicolumn{2}{|l|}{ Nivel de estudios } \\
\hline Primaria & $351(62,9 \%)$ \\
\hline Secundaria & $201(36,02 \%)$ \\
\hline Técnicos & $84(15,05 \%)$ \\
\hline Profesionales & $66(11,82 \%)$ \\
\hline \multicolumn{2}{|l|}{ Afiliación a seguridad social } \\
\hline Contributivo & $437(78,31 \%)$ \\
\hline Subsidiado & $121(21,68 \%)$ \\
\hline
\end{tabular}




Características sociodemográficas y en salud sexual y reproductiva de la población
de mujeres menopáusicas encuestadas Quindío (Colombia),
n

* Media \pm desviación estándar (DE)

La prevalencia del SGUM en el grupo estudiado fue del 51,61\% ( $\mathrm{n}=288 / 558)$. El síntoma más frecuente referido por las pacientes fue la sequedad vaginal (84\%), seguida por la dispareunia (57\%) (figura 1). En relación con los hallazgos de los síntomas vulvo-vaginales y del tracto urinario inferior relacionados con la presencia del SGUM, se encontró que un $71,87 \%$ de las mujeres sintomáticas presentó tres, un 22,91 \% presentó cuatro, y un 5,2\% presentó cinco o más síntomas.

La puntuación media total del IFSF en la población estudiada fue de 26,58; el puntaje mayor fue de 29,43 y el mínimo de 4,74, con una desviación estándar de $\pm 7,38$ puntos. La prevalencia de disfunción sexual en el grupo estudiado fue del 67,2\% $(\mathrm{n}=375 / 558)$, con una puntuación del IFSF de $19,47 \pm 8,52$. La disfunción sexual presente con más frecuencia fue el trastorno del deseo, con 375 casos (67,2\%), seguido por la alteración de la excitación con $252(45,16 \%)$ y, en tercer lugar, la alteración del orgasmo con 246 casos (44,08\%), seguido de alteración de la lubricación con 180 (32,25\%), y, finalmente, dolor durante el coito (dispareunia) con 78 casos (13,97\%). Se encontró que el 7,73\% presenta una disfunción sexual, el 78,13\% dos, y $14,13 \%$ tres o más disfunciones sexuales. En la tabla 2 se describe la puntuación detallada de cada dominio del IFSF en la población de mujeres con SGUM en el Quindío.

En el grupo de mujeres con SGUM, el 53,37\% $(n=155)$ consideraba que este impactaba de forma negativa en su vida diaria; el 64,23\% $(n=185)$ afirmó consecuencias negativas en su vida sexual, lo cual llevó a que el 57,98\% (n=167) de las mujeres afirmaran sentirse menos dispuestas al coito. Se encontró que al 27,43\% (n=79) les afectaba la 
Figura 1.

Síntomas del SGUM reportados por las mujeres menopáusicas en Quindío (Colombia), 2013-2016

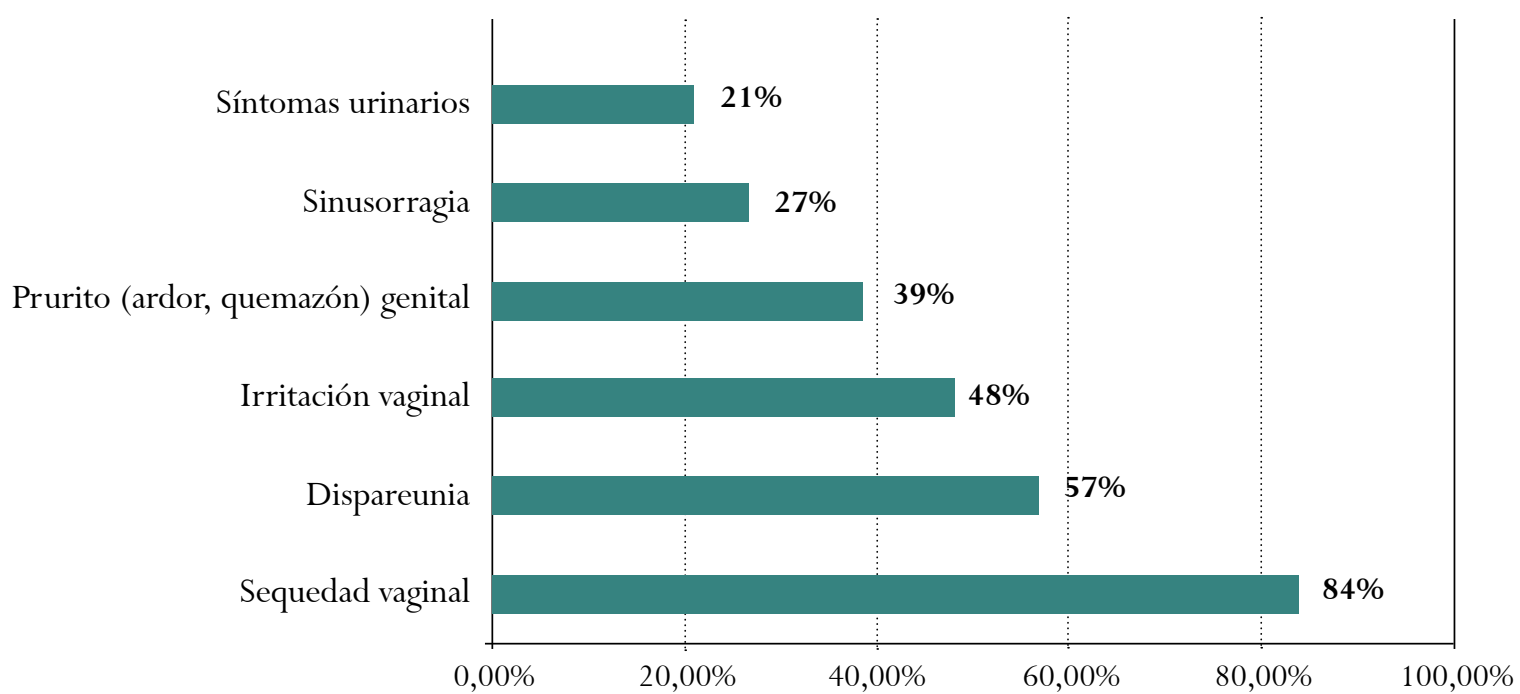

\begin{tabular}{|c|c|c|}
\hline \multicolumn{2}{|c|}{ Tabla 2.} \\
\hline \multicolumn{2}{|c|}{ Índice de función sexual femenina en mujeres del Quindío con SGUM } \\
\hline Dominios & Media $( \pm \mathrm{DE})$ & Disfunción sexual (\%) \\
\hline Deseo & $3,27 \pm 1,02$ & 67,2 \\
\hline Excitación & $3,57 \pm 1,08$ & 45,16 \\
\hline Lubricación & $3,72 \pm 1,14$ & 32,25 \\
\hline Orgasmo & $3,63 \pm 1,05$ & 44,08 \\
\hline Satisfacción & $4,08 \pm 1,17$ & 70,96 \\
\hline Dolor & $4,47 \pm 1,11$ & 13,97 \\
\hline
\end{tabular}

relación de pareja, observándose un deterioro en la autoestima en el 21,18\% $(n=61)$ de las mujeres. A pesar de estos porcentajes, no consideraron que el síntoma fuera lo suficientemente molesto como para la búsqueda de asistencia médica.

\section{DISCUSIÓN}

En esta investigación se encontró una prevalencia del 51,61\% del SGUM en el grupo estudiado; la sequedad vaginal representó el $84 \%$, seguida por la dispareunia, con el $57 \%$. Se encuentra además un significativo impacto en la sexualidad de este grupo de mujeres, que se caracteriza por una prevalencia del 67,2\% de disfunciones sexuales (deseo: 67,2\%, excitación: 45,16\%, orgasmo: 44,08\%, lubricación: 32,25\% y dolor: 13,97\%). De igual manera, se observó que es inusual la presencia de una sola disfunción sexual (7,73\%).

La prevalencia de SGUM en esta investigación es concordante con la descrita por otros autores $(10,12,15)$, pero inferior a lo reportado por Moral et al., que informan una prevalencia del $70 \%$ (16). 
Otros estudios muestran una prevalencia inferior en poblaciones similares que varía entre el 30,8\% (17) y $39 \%(18)$.

En relación con la sequedad vaginal como el síntoma más frecuente, nuestros resultados sobrepasan lo reportado en otros estudios, como el realizado por Chua et al. en Asia que informan una frecuencia de $57 \%$ (19), y otros que muestran una frecuencia de 27 a $55 \%$, realizados en poblaciones similares (20-23).

Respecto a la proporción de mujeres con tres de los síntomas vulvo-vaginales y del tracto urinario inferior, nuestros hallazgos son mayores $(71,87 \%)$ a los reportados por Pastore et al. (24), y a lo publicado por Espitia en una población similar de mujeres de la ciudad de Armenia (25).

En relación con la prevalencia de disfunción sexual, nuestros resultados también son superiores a los descritos previamente por nuestro grupo, con un 42,9\% (14), lo que podría estar relacionado con el hecho de que son mayoritariamente de bajo nivel de escolaridad y de estrato socioeconómico bajomedio, lo cual puede ser un factor que condiciona la existencia de desigualdades a la hora de solicitar la atención médica de forma oportuna, lo que agrava e incrementa la sintomatología.

Referente a los dominios de IFSF, la alteración del deseo sexual sobresalió como el trastorno más comúnmente reportado, superior a lo descrito en otra publicación colombiana $(38,4 \%)(8)$, lo cual podría explicarse por el tipo de población reclutada, la edad de las pacientes, así como por los criterios de inclusión del estudio. Al contrastar el impacto de los síntomas del SGUM en el funcionamiento sexual encontrado en esta investigación, con lo reportado por otros autores fuera del país, se observa que dicho impacto es igual de negativo al encontrado en mujeres españolas por Moral et al. (16), en mujeres europeas por Nappi et al. (26) y en Canadá por Labrie et al. (27).

En el estudio Vaginal Health: Insights Views \& Attitudes (VIVA) (28), le preguntaron online a un grupo de mujeres norteamericanas si el disconfort vaginal afectaba su vida, y el $80 \%$ respondió de forma afirmativa. El 75 \% manifestó consecuencias negativas en su vida sexual, $33 \%$ en la relación de pareja y el 26\% sobre la autoestima; porcentajes que resultan mayores a los reportados por nuestro estudio, pero no con menos impacto en la vida diaria y el desempeño sexual de las mujeres encuestadas. La diferencia entre los resultados obtenidos en esta investigación y los otros autores puede deberse a disparidades en aspectos raciales, a que mayoritariamente convivían en unión estable, a factores de índole sociocultural, a mayor edad de las mujeres, así como al tiempo de la menopausia.

En relación con los síntomas del SGUM y el impacto en la sexualidad, las mujeres con más síntomas presentaron un mayor porcentaje de trastornos sexuales, lo cual es similar a lo informado por Kagan et al. (29). Sin embargo, a pesar de la alta prevalencia de disfunciones sexuales, el impacto negativo en la sexualidad no se consideró como síntoma molesto que motivara la búsqueda de asistencia médica, lo cual muestra concordancia con otros estudios, donde solo un $25 \%$ de las mujeres buscaron ayuda debido a la sensación de vergüenza y a la creencia de que los síntomas eran consecuencia normal del envejecimiento $(28,30-32)$.

$\mathrm{Al}$ igual que en otras investigaciones, la sexualidad femenina se ve afectada de forma negativa por la menopausia $(8,19)$; así, se observa que el SGUM se relaciona con serias repercusiones en el deseo, el orgasmo e incremento del dolor, tal como lo confirmamos en este estudio.

Una de las limitaciones del estudio ha sido la selección de la muestra, que es no probabilística, y no permite la generalización; sin embargo, se logró una significativa población de mujeres al incluir al 90\% de la población disponible para la investigación. Entre las fortalezas se destaca que es el primer estudio de prevalencia del SGUM hecho en la región, y uno de los pocos trabajos realizados al respecto en población colombiana, además de ser el primero en haber considerado el impacto del SGUM en la salud sexual de las mujeres colombia- 
nas. La oportunidad de haberse realizado en una institución de tercer nivel le permite extrapolarse a toda la región.

\section{CONCLUSIONES}

Las mujeres estudiadas muestran una alta prevalencia del SGUM, cuyos síntomas afectan su sexualidad de forma negativa, ya que se asocia frecuentemente a disfunción sexual.

Se requieren estudios poblacionales, en la región, que describan la importancia del SGUM como problema de salud en nuestra población, a fin de definir la prioridad de establecer estrategias que impacten la calidad de vida de estas mujeres.

\section{AGRADECIMIENTOS}

Agradezco al doctor David Vásquez Awad, mi gran amigo y maestro, no solo por sus enseñanzas, sino por la riqueza de su conocimiento acerca de la menopausia y el climaterio, puesta al servicio de todos los interesados, sin cuyas observaciones no hubiese sido posible realizar esta investigación.

\section{FINANCIACIÓN}

En este estudio no se contó con el apoyo financiero de ninguna entidad, todo fue producto del esfuerzo económico del autor.

\section{REFERENCIAS}

1. Portman DJ, Gass ML. Vulvovaginal Atrophy Terminology Consensus Conference Panel. Genitourinary syndrome of menopause: New terminology for vulvovaginal atrophy from the International Society for the Study of Women's Sexual Health and the North American Menopause Society. Menopause. 2014;21:1063-8. https://doi. org/10.1097/GME.0000000000000329

2. Gandhi J, Chen A, Dagur G, Suh Y, Smith N, Cali $\mathrm{B}$, et al. Genitourinary syndrome of menopause: An overview of clinical manifestations, pathophysiology, etiology, evaluation and management. Am J Obstet Gynecol. 2016;215:704-11. https:// doi.org/10.1016/j.ajog.2016.07.045
3. Espitia-De La Hoz FJ, Orozco Gallego H. Abordaje diagnóstico y terapéutico del síndrome genitourinario en la menopausia; actualización. Rev Med UCR. 2017;117:67-84. https://doi. org/10.15517/rmucr.v11i2.34580

4. Nappi RE, Lachowsky M. Menopause and sexuality: Prevalence of symptoms and impact on quality of life. Maturitas. 2009;63:138-41. https://doi. org/10.1016/j.maturitas.2009.03.021

5. Cumming GP, Currie HD, Moncur R, Lee AJ. Web-based survey on the effect of menopause on women's libido in a computer-literate population. Menopause Int. 2009;15:8-12. https://doi. org/10.1258/mi.2009.009001

6. Espitia-De La Hoz FJ. Prevalence and characterization of sexual dysfunctions in women, in 12 Colombian cities, 2009-2016. Rev Colomb Obstet Ginecol. 2018;69:9-21. https://doi. org/10.18597/rcog.3035

7. Rosen R, Brown C, Heiman J, Leiblum S, Meston $\mathrm{C}$, Shabsigh R, et al. The Female Sexual Function Index (FSFI): A multidimensional self-report instrument for the assessment of female sexual function. J Sex Marital Ther. 2000;26:191-208. https://doi.org/10.1080/009262300278597

8. Vallejo-Medina P, Pérez-Durán C, Saavedra-Roa A. Translation, adaptation, and preliminary validation of the female sexual function index into Spanish (Colombia). Arch Sex Behav. 2017;1-14. https://doi.org/10.1007/s10508-017-0976-7

9. Palacios S, Mejía A, Neyro JL. Treatment of the genitourinary syndrome of menopause. Climacteric. 2015;18:23-9. https://doi.org/10.3109/13 697137.2015.1079100

10. Golmakani N, Parnan Emamverdikhan A, Zarifian A, Sajadi Tabassi SA, Hassanzadeh M. Vitamin E as alternative local treatment in genitourinary syndrome of menopause: A randomized controlled trial. Int Urogynecol J. 2018. https://doi.org/10.1007/ s00192-018-3698-z

11. Portman D, Palacios S, Nappi RE, Mueck AO. Ospemifene, a non-oestrogen selective oestrogen receptor modulator for the treatment of vaginal 
dryness associated with postmenopausal vulvar and vaginal atrophy: A randomised, placebo-controlled, phase III trial. Maturitas. 2014;78:91-8. https://doi.org/10.1016/j.maturitas.2014.02.015

12. Bruyniks N, Nappi RE, Castelo-Branco C, de Villiers TJ, Simon J. Effect of ospemifene on moderate or severe symptoms of vulvar and vaginal atrophy. Climacteric. 2016;19:60-5. https://doi. org/10.3109/13697137.2015.1113517

13. Rabley A, O’Shea T, Terry R, Byun S, Louis Moy M. Laser Therapy for Genitourinary Syndrome of Menopause. Curr Urol Rep. 2018;19:83. https:// doi.org/10.1007/s11934-018-0831-y

14. Espitia-De La Hoz FJ. Prevalencia de disfunción sexual en mujeres del Eje Cafetero. Rev Cienc Biomed. 2016;7:15-23.

15. Nappi RE, Kingsberg S, Maamari R, Simon J. The CLOSER (CLarifying Vaginal Atrophy's Impact On SEx and Relationships) survey: Implications of vaginal discomfort in postmenopausal women and in male partners. J Sex Med. 2013;10:2232-41. https://doi. org/10.1111/jsm.12235

16. Moral E, Delgado JL, Carmona F, Caballero B, Guillán C, González PM, et al., as the writing group of the GENISSE study. Genitourinary syndrome of menopause. Prevalence and quality of life in Spanish postmenopausal women. The GENISSE study. Climacteric. 2018;21:167-73. https://doi.org/10.10 80/13697137.2017.1421921

17. Geng L, Zheng Y, Zhou Y, Li C, Tao M. The prevalence and determinants of genitourinary syndrome of menopause in Chinese mid-life women: A singlecenter study. Climacteric. 2018;21:478-82. https:// doi.org/10.1080/13697137.2018.1458832

18. Nappi RE, Kokot-Kierepa M. Women's voices in the menopause: Results from an international survey on vaginal atrophy. Maturitas. 2010;67:233-8. https:// doi.org/10.1016/j.maturitas.2010.08.001

19. Chua Y, Limpaphayom KK, Cheng B, Ho CM, Sumapradja K, Altomare C, et al. Genitourinary syndrome of menopause in five Asian countries: Results from the Pan-Asian REVIVE survey. Climacteric.
2017;20:367-73. https://doi.org/10.1080/1369713 7.2017 .1315091

20. van Geelen JM, van de Weijer PH, Arnolds HT. Urogenital symptoms and resulting discomfort in non-institutionalized Dutch women aged 50-75 years. Int Urogynecol J Pelvic J. 2000;11:9-4. https://doi. org/10.1007/PL00004023

21. Hutchinson-Colas J, Segal S. Genitourinary syndrome of menopause and the use of laser therapy. Maturitas. 2015;82:342-5. https://doi.org/10.1016/j. maturitas.2015.08.001

22. Jackson SL, Boyko EJ, Scholes D, Abraham L, Gupta K, Fihn SD. Predictors of urinary tract infection after menopause: A prospective study. Am J Med. 2004;117:903-11. https://doi.org/10.1016/j. amjmed.2004.07.045

23. Oskay UY, Beji NK, Yalcin O. A study on urogenital complaints of postmenopausal women aged 50 and over. Acta Obstet Gynecol Scand. 2005;84:72-8. https://doi.org/10.1111/j.0001-6349.2005.00645.x

24. Pastore LM, Carter RA, Hulka BS, Wells E. Selfreported urogenital symptoms in postmenopausal women: Women's Health Initiative. Maturitas. 2004;10:292-303. https://doi.org/10.1016/j. maturitas.2004.06.019

25. Espitia-De La Hoz FJ, Orozco-Gallego H. Estriol vs estrógenos conjugados de origen equino en el tratamiento del síndrome genitourinario de la menopausia. Ginecol Obstet Mex. 2018;86:117-126. https://doi.org/10.24245/gom.v86i2.1881

26. Nappi RE, Palacios S, Panay N, Particco M, Krychman ML. Vulvar and vaginal atrophy in four European countries: Evidence from the European REVIVE Survey. Climacteric. 2016;19:188-97. https://doi.or g/10.3109/13697137.2015.1107039

27. Labrie F, Archer DF, Koltun W, Vachon A, Young D, Frenette L, et al. Efficacy of intravaginal dehydroepiandrosterone (DHEA) on moderate to severe dyspareunia and vaginal dryness, symptoms of vulvovaginal atrophy, and of the genitourinary syndrome of menopause. Menopause. 2016;23:243-56. https:// doi.org/10.1097/GME.0000000000000571 
28. Nappi RE, Kokot-Kierepa M. Vaginal Health: Insights, Views \& Attitudes (VIVA) - Results from an international survey. Climacteric. 2012;15:36-44. https://doi.org/10.3109/13697137.2011.647840

29. Kagan R, Rivera E. Restoring vaginal function in postmenopausal women with genitourinary syndrome of menopause. Menopause. 2018;25:106-8. https:// doi.org/10.1097/GME.0000000000000958

30. Ibe C, Simon JA. Vulvovaginal atrophy: Current and future therapies (CME). J Sex Med. 2010;7:1042-50. https://doi.org/10.1111/j.1743-6109.2009.01692.x
31. Huang KE. Menopause perspectives and treatment of Asian women. Semin Reprod Med. 2010;28:396403. https://doi.org/10.1055/s-0030-1262899

32. Espitia-De La Hoz FJ, Orozco Gallego H, Echeverri Ocampo LM. Terapia hormonal y no hormonal en la vaginitis atrófica posmenopáusica: cura y satisfacción a mediano y a largo plazo de los síntomas. Rev Col Men. 2016;22:8-17. 